\title{
Anisotropic high-field diffusion of holes in silicon
}

\author{
J. M. Hinckley a) and J. Singh \\ Department of Electrical Engineering and Computer Science, The University of Michigan, \\ Ann Arbor, Michigan 48109-2122
}

(Received 9 November 1994; accepted for publication 3 March 1995)

\begin{abstract}
Anisotropy of the silicon valence band leads to a strong dependence of charge carrier transport properties, upon the orientation of the electric field. A detailed anisotropic Monte Carlo method has been applied to the calculation of the hole diffusion coefficient in silicon, studying its dependence on field magnitude and orientation. The longitudinal diffusion coefficient is found to have a dependence on the field orientation which is similar in degree to the more familiar dependence of the drift velocity on field orientation. However, it is found that the transverse diffusion coefficient has a substantially stronger dependence on field orientation. At the highest field which has been studied, $50 \mathrm{kV} / \mathrm{cm}$, the transverse diffusion coefficient almost doubles as one shifts from a field

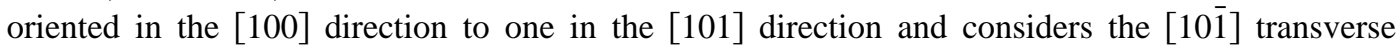
direction. (C) 1995 American Institute of Physics.
\end{abstract}

The valence band structure of essentially all semiconductors is highly anisotropic. While this anisotropy manifests itself in physical observations under strong magnetic fields (e.g., cyclotron resonance experiments), its influence on nonmagnetotransport related phenomena is usually minimal. For example, the hole drift velocity has a rather small anisotropy. ${ }^{1}$ This is due to the fact that during drift, a typical hole scatters over a large phase space, and thus samples the band structure along all directions. Due to the relative ease of doing transport experiments as compared to cyclotron resonance measurements (where very strong magnetic fields and low temperatures are needed), it would be very useful to identify some transport related effect where the band structure anisotropy manifests itself in a manner stronger than it does in drift velocity.

Compared to the drift velocity, the behavior of high-field hole diffusivity is less well known. Measurements have been made both by noise ${ }^{2}$ and time-of-flight ${ }^{2,3}$ techniques. The only reported values of room-temperature hole data in silicon, are for the longitudinal diffusivity with the electric field in the [111] direction. Further investigation of the hole diffusivity, both longitudinal and transverse, for several field orientations, should prove useful for a more accurate understanding of charge carrier transport and noise in silicon devices, as well as giving additional insight into the physics arising from valence band anisotropy.

In the present work, we have extended our Monte Carlo $\operatorname{method}^{4}$ to examine the behavior of both the longitudinal and the transverse diffusion coefficient of holes in silicon for different directions of high electric field. The calculational method accounts for the anisotropy of the valence band by using the $6 \times 6 \mathrm{Kohn}-$ Luttinger Hamiltonian, with spin-orbit splitting, to model the valence band. This gives both anisotropic energy values, as well as eigenvectors which are used in the calculation of scattering rates, that are themselves anisotropic. The scattering mechanisms included in the calculations are acoustic and nonpolar optical phonon scattering,

${ }^{a)}$ Electronic mail: ncko@caen.engin.umich.edu at room temperature. Thus, we are considering hole transport in pure materials only.

For a given field orientation, the Monte Carlo calculates the velocity fluctuation autocorrelation function tensors, $C_{i j}, i, j=x, y, z .{ }^{5}$ For example, $C_{x x}$ is shown in Fig. 1, for $E=0 \mathrm{~V} / \mathrm{cm}$ and $50 \mathrm{kV} / \mathrm{cm}$, in the [100] direction. The integral of this with respect to time gives the corresponding diffusion coefficient tensor: ${ }^{1}$

$$
D_{i j}=\int_{0}^{\infty} C_{i j}(t) d t
$$

The projection of the diffusion coefficient tensor in the direction of the electric field gives the longitudinal diffusion coefficient, while projection on a direction perpendicular to the field gives the transverse diffusion coefficient. In this work, fields oriented in three different directions were used: [100], [111], and [101]. For electric fields in the [100] and [111] directions, all directions which are perpendicular to the electric field are equivalent, having the same transverse dif-

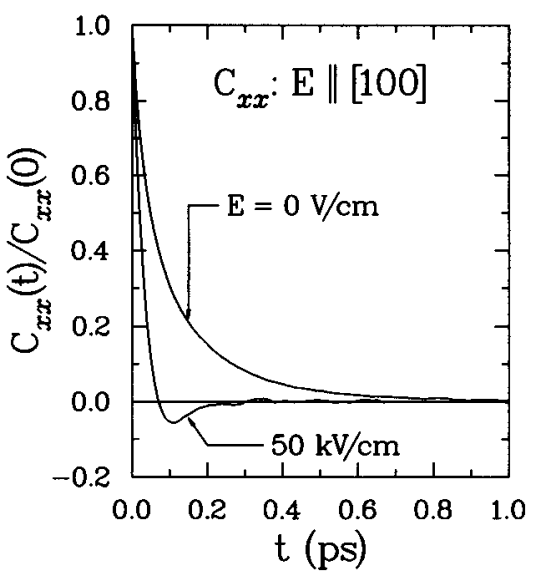

FIG. 1. Example of the velocity fluctuation autocorrelation function, from which the diffusivity is calculated in this work. The component $C_{x x}$ is shown here, for both zero electric field and an electric field of $50 \mathrm{kV} / \mathrm{cm}$ in the [100] direction. The corresponding diffusion coefficient tensor element, $D_{x x}$, is calculated as the integral of $C_{x x}$ with respect to time. 
fusion coefficient. This is consequence of the symmetry of the system. However, when the field is oriented in the [101] direction, there are two nonequivalent perpendicular directions: [010] and [101] . These can be seen to be symmetrically distinct and our results show that they correspond to two very distinct values of transverse diffusion coefficients.

The specific relations between the Cartesian components of the diffusion tensor and the longitudinal and transverse diffusivity depend on the field orientation, and are as follows. For a [100] field:

$$
\begin{aligned}
& D_{l}=D_{x x} \\
& D_{t}=D_{y y}=D_{z z} \\
& D_{x y}=D_{y z}=D_{z x}=0 .
\end{aligned}
$$

For a [111] field:

$$
\begin{aligned}
& D_{a} \equiv D_{x x}=D_{y y}=D_{z z} \\
& D_{b} \equiv D_{x y}=D_{y z}=D_{z x} \\
& D_{l}=D_{a}+2 D_{b} \\
& D_{t}=D_{a}-D_{b} .
\end{aligned}
$$

For a [101] field:

$$
\begin{aligned}
& D_{a} \equiv D_{x x}=D_{z z} \\
& D_{b} \equiv D_{z x} \\
& D_{x y}=D_{y z}=0 \\
& D_{l}=D_{a}+D_{b} \\
& D_{t 1}=D_{y y} \\
& D_{t 2}=D_{a}-D_{b} \\
& D_{t}=\cos ^{2} \phi D_{t 1}+\sin ^{2} \phi D_{t 2},
\end{aligned}
$$

where $\phi$ is the angle between the $y$ axis and any chosen arbitary direction perpendicular to [101].

Figure 2 shows the results of our calculations of the hole diffusion coefficient in silicon at room temperature. For each value and orientation of electric field, a calculation involved the simulation of $2 \times 10^{5}$ particles over a 2 ps time of flight. At room temperature, this is sufficient time for the simulated particles to lose all correlation with their initial conditions, as shown by the example in Fig. 1. Also shown in Fig. 2 are discrete points from the measured data of Reggiani, et al. ${ }^{2}$ These are similar to other room-temeprature measurements found in the literature ${ }^{3,6}$ all being for the longitudinal diffusivity with a field in the [111] direction. The corresponding data from our calculations are highlighted as the dashed curve in Fig. 2. The difference between the measurements and our calculations is small, and may be attributed to the omission of impurity scattering in our calculations.

For the longitudinal diffusion coefficient, it is seen that the value for a field oriented in the [100] direction is somewhat larger than values for fields in either the [111] or [101] directions. As in the case of drift velocity, this is a consequence of the greater heavy hole band curvature in the [100] direction than in the other directions.

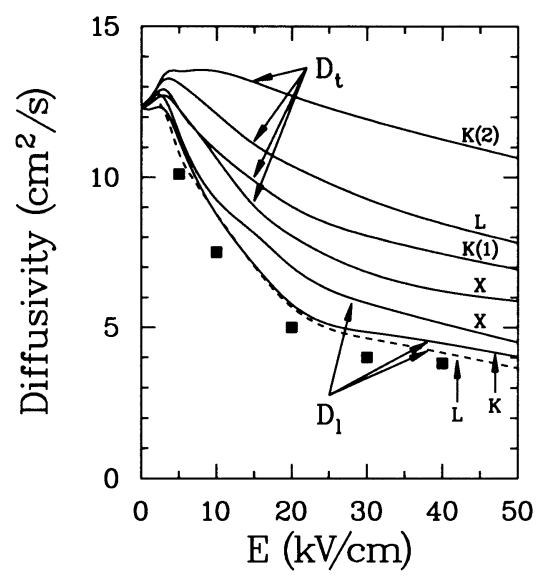

FIG. 2. Diffusivity of holes in silicon. Upper four curves are transverse diffusivity $\left(D_{t}\right)$; lower three curves are longitudinal diffusivity $\left(D_{l}\right)$. Labeling X, L, K refers to the electric field orientation: X: [100]; L: [111]; K: [101]. For the [101] field orientation, the two non-equivalent transverse directions are [010], labeled K(1), and [101]], labeled K(2). Representative measured data from Ref. 9 are shown at discrete points. These data are for $D_{l}$ with the field in the [111] direction. This corresponds to the dashed curve calculated in this work.

Also, it will be noted from the data shown in Fig. 2, that all the transverse diffusion coefficient values are greater than the longitudinal diffusion coefficient values. This is the same qualitative behavior that was explained in Ref. 7 for electrons, and is due to the fact that carriers spreading in the direction of the field have an increased likelihood of scattering, which tends to suppress that spreading, whereas this is not the case for transverse spreading.

Compared to the longitudinal diffusion coefficient, the transverse coefficient values are shown to be much more sensitive to the direction of the electric field. The effect becomes more pronounced as the magnitude of the field is increased. One of the most striking features of these data is that orienting the electric field in the [101] direction gives rise to three very distinct values of diffusion coefficient, one longitudinal and two transverse ones. At an electric field of $50 \mathrm{kV} / \mathrm{cm}$, the longitudinal diffusion coefficient, $D_{l}$ is calculated to be $4.0 \mathrm{~cm}^{2} / \mathrm{s}$, while the transverse coefficient in the [010] direction $D_{t 1}$ is $6.9 \mathrm{~cm}^{2} / \mathrm{s}$ and the transverse coefficient in the [101] direction $D_{t 2}$ is $10.4 \mathrm{~cm}^{2} / \mathrm{s}$.

Our initial investigations of this matter indicate that the hole diffusion cloud may have a complex, nonelliptical shape, resulting from the anisotropic band structure. We find that for very short times, definite preferential directions are imposed on the motion of the holes, aside from any bias imposed by an electric field. The diffusion cloud is similar in shape to the heavy hole constant energy surface. The same effect is found at a high electric field, although somewhat obscured by the effects of the field. We expect that the anisotropy of the diffusivity is a consequence of the nonellipticity of the diffusion cloud and are continuing our research on this matter.

The results of this work show two features of the hole diffusion coefficient which depend on the anisotropy of the valence band. First, for a given field magnitude, the degree of difference between the longitudinal and the transverse dif- 
fusion coefficients depends on the field orientation. This difference is greater for fields in the [111] and [101] directions than for fields in the [100] direction. Second, for fields in the [101] directions, there are two symmetrically nonequivalent transverse directions. The transverse diffusion coefficients in these directions, $D_{t 1}$ and $D_{t 2}$, are not only clearly distinct from the longitudinal coefficient $D_{l}$, but are also quite distinct from each other. The anisotropy of the valence band therefore gives rise to a complex structure in the diffusion coefficient.

Direct application of this knowledge should increase the accuracy of silicon device modeling. The data presented here show that published high field hole diffusivities represent the lower limit of $D_{l}$. Inverting the problem, analysis of longitudinal and transverse thermal noise data, to which diffusion is related through the Nyquist relation, ${ }^{8}$ could yield information about the bandstructure anisotropy of novel semiconduc- tors, such as diamond, which are not readily analyzed with conventional methods, such as magnetotransport.

This work was supported by the U.S. Air Force (Grant No. AFOSR-91-0349) and by the U.S. Army URI Program (DAAL03-92-G-0109).

${ }^{1}$ C. Jacoboni and L. Reggiani, Rev. Mod. Phys. 55, 645 (1983).

${ }^{2}$ L. Reggiani, Phys. Nonlinear Trans. Semicond. NATO ASI, 52, 467 (1980).

${ }^{3}$ T. W. Sigmon and J. F. Gibbons, Appl. Phys. Lett. 15, 320 (1969).

${ }^{4}$ J. M. Hinckley, Ph.D. thesis, University of Michigan, Ann Arbor, 1990.

${ }^{5}$ J. M. Hinckley and J. Singh, Phys. Rev. B (to be published).

${ }^{6}$ F. Nava, C. Canali,L. Reggiani, D. Gasquet, J. C. Vaissiere,and J. P. Nougier, J. Appl. Phys. 50, 922 (1979).

${ }^{7}$ R. Brunetti,C. Jacoboni, F. Nava, L. Reggiani, G. Bosman, and R. J. J. Zijlstra, J. Appl. Phys. 52, 6713 (1981).

${ }^{8}$ Y. K. Pozhela, Hot-Electron Transport in Semiconductors, edited by L. Reggiani (Springer, Berlin, 1984), Chap. 4.

${ }^{9}$ L. Reggiani, J. Phy. Soc. Jpn. Suppl. 49, 317 (1980). 